Article

\title{
Consecutive-Day Ventricular and Atrial Cardiomyocyte Isolations from the Same Heart: Shifting the Cost-Benefit Balance of Cardiac Primary Cell Research
}

\author{
Joachim Greiner ${ }^{1,+}$ D , Teresa Schiatti ${ }^{1,+}$, Wenzel Kaltenbacher ${ }^{1,+}{ }^{+}$, Marica Dente ${ }^{2}$, Alina Semenjakin ${ }^{1}$ (D, \\ Thomas Kok ${ }^{1}$, Dominik J. Fiegle ${ }^{3}$, Thomas Seidel ${ }^{3}$, Ursula Ravens ${ }^{1}$ (D) Peter Kohl 1,4 ${ }^{\text {, Rémi Peyronnet }}{ }^{1, *,+}$ \\ and Eva A. Rog-Zielinska ${ }^{1, *, \dagger}$
}

Citation: Greiner, J.; Schiatti, T.; Kaltenbacher, W.; Dente, M.;

Semenjakin, A.; Kok, T.; Fiegle, D.J.; Seidel, T.; Ravens, U.; Kohl, P.; et al. Consecutive-Day Ventricular and Atrial Cardiomyocyte Isolations from the Same Heart: Shifting the Cost-Benefit Balance of Cardiac Primary Cell Research. Cells 2022, 11, 233. https://doi.org/10.3390/ cells11020233

Academic Editors: Lucio Barile

Received: 8 November 2021

Accepted: 5 January 2022

Published: 11 January 2022

Publisher's Note: MDPI stays neutral with regard to jurisdictional claims in published maps and institutional affiliations.

Copyright: (C) 2022 by the authors. Licensee MDPI, Basel, Switzerland. This article is an open access article distributed under the terms and conditions of the Creative Commons Attribution (CC BY) license (https:// creativecommons.org/licenses/by/ $4.0 /)$.
1 Institute for Experimental Cardiovascular Medicine, University Heart Center Freiburg-Bad Krozingen and Faculty of Medicine, Albert-Ludwig University of Freiburg, 79110 Freiburg im Breisgau, Germany; joachim.greiner@uniklinik-freiburg.de (J.G.); teresa.schiatti@uniklinik-freiburg.de (T.S.); david.kaltenbacher@uniklinik-freiburg.de (W.K.); alina07@gmx.de (A.S.); thomas.kok@uniklinik-freiburg.de (T.K.); ursula.ravens@uniklinik-freiburg.de (U.R.); peter.kohl@uniklinik-freiburg.de (P.K.)

2 Department of Experimental and Clinical Medicine, Division of Physiology, University of Florence, 50134 Florence, Italy; marica.dente@unifi.it

3 Institute of Cellular and Molecular Physiology, Friedrich-Alexander-University of Erlangen-Nürnberg, 91054 Erlangen, Germany; dominik.fiegle@fau.de (D.J.F.); thomas.seidel@fau.de (T.S.)

4 CIBSS Centre for Integrative Biological Signalling Studies, University of Freiburg, 79110 Freiburg im Breisgau, Germany

* Correspondence: remi.peyronnet@uniklinik-freiburg.de (R.P.); eva.rog-zielinska@uniklinik-freiburg.de (E.A.R.-Z.)

+ These authors contributed equally to this work.

Abstract: Freshly isolated primary cardiomyocytes (CM) are indispensable for cardiac research. Experimental CM research is generally incompatible with life of the donor animal, while human heart samples are usually small and scarce. CM isolation from animal hearts, traditionally performed by coronary artery perfusion of enzymes, liberates millions of cells from the heart. However, due to progressive cell remodeling following isolation, freshly isolated primary CM need to be used within 4-8 h post-isolation for most functional assays, meaning that the majority of cells is essentially wasted. In addition, coronary perfusion-based isolation cannot easily be applied to human tissue biopsies, and it does not straightforwardly allow for assessment of regional differences in CM function within the same heart. Here, we provide a method of multi-day CM isolation from one animal heart, yielding calcium-tolerant ventricular and atrial CM. This is based on cell isolation from cardiac tissue slices following repeated (usually overnight) storage of the tissue under conditions that prolong CM viability beyond the day of organ excision by two additional days. The maintenance of cells in their near-native microenvironment slows the otherwise rapid structural and functional decline seen in isolated CM during attempts for prolonged storage or culture. Multi-day slice-based CM isolation increases the amount of useful information gained per animal heart, improving reproducibility and reducing the number of experimental animals required in basic cardiac research. It also opens the doors to novel experimental designs, including exploring same-heart regional differences.

Keywords: cardiomyocyte isolation; single cell; sarcomere shortening; tissue preservation

\section{Introduction}

Single cells, isolated from various tissues, have been a foundation for the development of mechanistic insight into many organ systems, promoting the understanding of physiology, pathology, and therapeutic interventions. Specifically, in experimental cardiac research, $\mathrm{CM}$ isolated from human and animal hearts have become indispensable over the 50 years since the first description of Langendorff perfusion-based enzymatic CM isolation [1]. 
In general, primary adult $\mathrm{CM}$ isolation is performed after sacrificing the experimental animal. While every successful isolation yields millions of $\mathrm{CM}$, most of these cells can be used for functional research only over a relatively short time period. Because most commonly employed functional CM assays (such as electrophysiology, functional imaging, or mechanics measurements) are low-throughput, the vast majority of isolated viable $\mathrm{CM}$ are usually discarded. Therefore, for each set of single CM experiments, an animal is sacrificed, although each heart contains enough CM for tens, if not hundreds, of functional experiments.

Such wastefulness arises additionally from the fact that the quality of freshly isolated $\mathrm{CM}$ declines swiftly after liberation from tissue. Adverse structural and functional remodeling can be seen as early as $4-8 \mathrm{~h}$ post-isolation. The detrimental changes observed in $\mathrm{CM}$ within hours of isolation include CM swelling and deformation [2-4], changes in wholecell capacitance [5], remodeling of trans-sarcolemmal and intracellular ion handling [6-8], depressed electro-mechanical function [9], internalization of surface proteins [10], loss of caveolae and transverse tubules [7], myofilament atrophy [3], or mitochondrial swelling and rupture $[2,5]$. The factors contributing to the steep decline in quality of freshly isolated $\mathrm{CM}$ are thought to include the elimination of mechanical support structures (cell-cell and cell-matrix coupling), the elimination of paracrine signaling, and the cessation of electrical stimulation and mechanically loaded contractions. This progressive cell remodeling curtails the relevance of culturing freshly isolated $\mathrm{CM}$ as a reproducible and representative experimental model system. In contrast, while immortalized or stem cell-derived cardiac cell lines are commercially available and sustainable in culture, they display an immature phenotype lacking homology to many structural and functional features of adult CM [11].

Here we develop and validate a method of extending both atrial and ventricular cell-in-tissue lifetime and quality by conducting multi-day CM isolations from the same rabbit heart. We show that live tissue storage [12] can be combined with slice-based CM isolation [13] to yield CM from the same donor heart over multiple days, circumventing the adverse remodeling in transcript levels, microscopic structure, and contraction and relaxation kinetics that are otherwise seen in isolated CM over the same period of time. Our method offers new opportunities for the dissection of regional differences in CM properties, and it may be applied to tissue that cannot be perfused (i.e., human or large animal heart biopsies). Multi-day CM isolation therefore offers a means towards extending the time period over which high-quality single-cell research can be conducted on adult primary CM, while at the same time lowering intra-individual variability and contributing to the aim of reducing the number of animals needed for experimental research.

\section{Materials and Methods}

\subsection{Animals}

All experiments were carried out according to the guidelines stated in Directive 2010/63/EU of the European Parliament on the protection of animals used for scientific purposes and were approved by the local authorities in Baden-Württemberg (X$16 / 10 R)$. New Zealand white rabbits were used in this study ( $n=18$, age $\approx 10$ weeks, both sexes, weight $\approx 1700 \mathrm{~g}$ ). Rabbits were anesthetized via intramuscular injection of esketamine hydrochloride (Ketanest S $25 \mathrm{mg} / \mathrm{mL}$, Pfizer Pharma PFE GmbH, Berlin, Germany; $12.5 \mathrm{mg} / \mathrm{kg}$ body weight) and xylazine hydrochloride (Rompun $2 \%$, Bayer Vital $\mathrm{GmbH}$, Leverkusen, Germany; $0.2 \mathrm{~mL} / \mathrm{kg}$ body weight). During anesthesia, 1000 units of heparin (Heparin Sodium 5000 I.U./mL, B. Braun Melsungen AG, Melsungen, Germany) and $5 \mathrm{mg}$ of esketamine hydrochloride were given intravenously. Thiopental (Thiopental Inresa $0.5 \mathrm{~g}$, Inresa Arzneimittel GmbH, Freiburg, Germany; $12.5 \mathrm{mg} / \mathrm{mL}$ ) was then injected intravenously until apnea.

\subsection{Cardiac Slice Preparation}

An overview of the protocol steps is provided in Figure 1. After excision, the heart was Langendorff perfused for $\sim 1$ min (until cessation of contractions) with warm $\left(37^{\circ} \mathrm{C}\right)$ modi- 
fied Tyrode's solution (flow rate $20 \mathrm{~mL} / \mathrm{min}$ ) containing (in $\mathrm{mM}$ ): $138 \mathrm{NaCl}, 0.33 \mathrm{NaH}_{2} \mathrm{PO}_{4}$, $5.4 \mathrm{KCl}, 2 \mathrm{MgCl}_{2}, 10$ HEPES, 10 glucose, $0.5 \mathrm{CaCl}_{2}$, and 30 2,3-butanedione monoxide; $\mathrm{pH}$ 7.3, $300 \mathrm{mOsm}$. This step can be omitted when processing tissue that cannot be perfused.

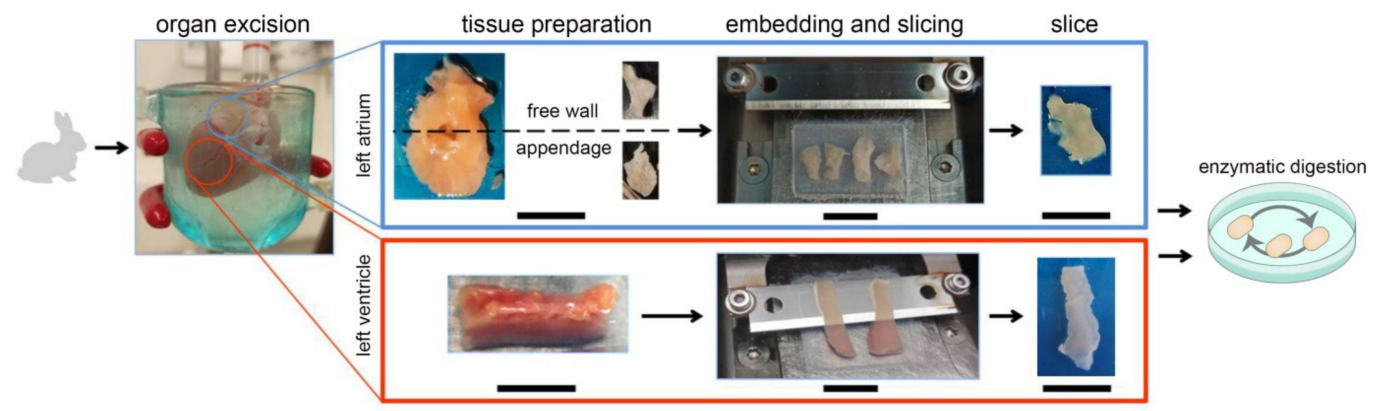

Figure 1. Overview of the protocol for the preparation of tissue slices and CM isolation from rabbit left atrium (top) and left ventricle (bottom), scale bars $10 \mathrm{~mm}$.

The heart was then dissected in cold $\left(4^{\circ} \mathrm{C}\right)$ modified Tyrode's solution; the left ventricular free wall, left atrial free wall, and left atrial appendage were used for further processing. The tissue was cut into blocks with an X/Y size of up to $1 \times 1 \mathrm{~cm}^{2}$ (ventricle) $/ 0.5 \times 0.5 \mathrm{~cm}^{2}$ (atria). These were embedded in $4 \%$ low melting point agarose (Carl Roth, Karlsruhe, Germany) prepared with modified Tyrode's solution, cooled to $4{ }^{\circ} \mathrm{C}$, and mounted on the stage of a vibratome (7000-smz-2 with 7550-1-SS blade, both Campden Instruments Ltd., Loughborough, UK) using cyanoacrylate-based low-viscosity glue. During mounting, care was taken to visually estimate the prevailing orientation of CM within the slice, and to mount the tissue so that the cutting plane would be parallel to the epicardial surface of the tissue block. The tissue was submerged in ice-cold modified Tyrode's solution bubbled with $\mathrm{O}_{2}$, and sliced into $300 \mu \mathrm{m}$ thick sections at a vertical blade vibration frequency of $60 \mathrm{~Hz}$ (ventricle) and $80 \mathrm{~Hz}$ (atria), an amplitude of $1.5 \mathrm{~mm}$, and an advancement velocity of $40 \mu \mathrm{m} / \mathrm{s}$ (ventricle) and $10 \mu \mathrm{m} / \mathrm{s}$ (atria). Ventricular tissue was freshly sliced from tissue chunks on each day before CM isolation, whereas atrial tissue was sliced on the day of heart excision and the slices were stored until CM isolation. All tissue was stored in modified Tyrode's solution at $4{ }^{\circ} \mathrm{C}$ and under room atmosphere, for up to three days.

\subsection{Cell Isolation}

CM isolation from slices was adapted based on a previously published protocol [13]. In brief, the agarose around the slice was gently removed, and the slices were placed in 35 $\mathrm{mm}$ diameter plastic Petri dishes and washed in modified Tyrode's solution. Slices were brought up to room temperature, then rinsed with wash solution 1 containing (in $\mathrm{mM}$ ): $100 \mathrm{NaCl}, 2.5 \mathrm{KH}_{2} \mathrm{PO}_{4}, 15 \mathrm{KCl}, 2 \mathrm{MgCl}_{2}, 10 \mathrm{HEPES}, 10$ glucose, 20 taurine, 20 L-glutamic acid monopotassium salt, and 30 2,3-butanedione monoxide (BDM), supplemented with

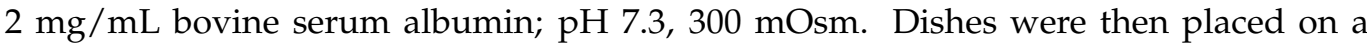
custom-built heated orbital shaker. All isolation steps were performed with constant rocking at $65 \mathrm{rpm}$, and at $37^{\circ} \mathrm{C}$. Tissue was digested for $12 \mathrm{~min}$ using proteinase mix XXIV (concentration: $0.5 \mathrm{mg} / \mathrm{mL}$ in $2 \mathrm{~mL}$ wash solution 1; Sigma-Aldrich, St. Louis, MO, USA). Tissue was then rinsed twice in wash solution 1 (each $2 \mathrm{~mL}$ ), and further digested with Liberase TL Research Grade (concentration: $2.5 \mathrm{mg} / \mathrm{mL}$ in $2 \mathrm{~mL}$ wash solution 1 supplemented with $5 \mu \mathrm{M} \mathrm{CaCl}_{2}$; Hoffmann-La Roche, Basel, Switzerland) for up to $45 \mathrm{~min}$ (ventricles)/15 min (atria). Tissue was then transferred into $2 \mathrm{~mL}$ of wash solution 2 (the same as wash solution 1, but with bovine serum albumin content increased to $10 \mathrm{mg} / \mathrm{mL}$, and supplemented with $5 \mu \mathrm{M} \mathrm{CaCl}_{2}$ ) and gently dissociated mechanically, using forceps to free individual CM. Calcium recovery and partial BDM washout were done by a stepwise (usually in 9 steps) addition of modified recording Tyrode's solution containing (in $\mathrm{mM}$ ): $137 \mathrm{NaCl}, 4 \mathrm{KCl}, 1 \mathrm{MgCl}_{2}, 10 \mathrm{HEPES}, 10$ glucose, $1.8 \mathrm{CaCl}_{2}$, 20 taurine, 10 creatine, 5 adenosine, and 2 L-carnitine; pH 7.3, 300 mOsm. This was continued until 
a final concentration of $1 \mathrm{mM} \mathrm{Ca}^{2+}$ and $12.8 \mathrm{mM}$ BDM were reached. The time interval between steps was $2 \mathrm{~min}$ (ventricles) and $5 \mathrm{~min}$ (atria).

The cell suspension was then filtered through mesh (filter size: $1 \mathrm{~mm} \times 1 \mathrm{~mm}$ ) and CM were allowed to settle for $15 \mathrm{~min}$ in a $15 \mathrm{~mL}$ centrifuge tube. The supernatant was removed, and the CM were re-suspended in modified recording Tyrode's solution (removing residual $\mathrm{BDM}$ and bringing the $\mathrm{Ca}^{2+}$ concentration to $1.8 \mathrm{mM}$ ). Composition of all solutions used for CM isolation can be found in Table S1 in Supplementary Materials.

\subsection{Assessment of Cell Isolation Yields}

Live $\mathrm{CM}$ were imaged post-isolation using a Leitz Laborlux 11 microscope (Leica Microsystems, Vienna, Austria) with a $2.5 \times$ air objective and an eyepiece camera (MikrOkular, Bresser, Rhede, Germany). To assess the yield of rod-shaped CM, we counted rod-shaped and dead (rounded) CM in at least three fields of view per isolation (60-500 CM manually counted per field of view). To assess contractile function, we paced CM in a perfusion chamber equipped with field stimulation electrodes (RC-27N, Warner Instruments, Hamden, MA, USA), driven by a MyoPacer field stimulator (applied voltage was twice the threshold and maximally $12 \mathrm{~V}$, pulse duration $5 \mathrm{~ms}$; pacing frequency was $0.5 \mathrm{~Hz}$ for ventricles and $1 \mathrm{~Hz}$ for atria; IonOptix, Westwood, MA, USA). Fractions of rod-shaped and contracting CM (i.e., responding to field stimulation without arrhythmic events, e.g., pauses, extra contractions) are expressed as percent of all CM in a field of view.

\subsection{High-Resolution Imaging}

To assess the regularity of cross-striations and the potential presence of membrane 'blebbing' (evidence of local detachment of the membrane from the cytoskeleton), highresolution imaging was performed using a Leica DMi8 microscope with a $60 \times$ oil objective for the ventricular CM, and with a Leica TCS SP8 X laser scanning confocal microscope (both Leica Microsystems, Vienna, Austria) using a $40 \times$ water immersion objective.

\subsection{Sarcomere Shortening Dynamics}

Unloaded CM shortening was assessed optically, as described previously [14]. Briefly, contractions were triggered by field stimulation, as described above, at room temperature. $\mathrm{CM}$ were imaged using an inverted microscope with a $40 \times$ air objective using phase contrast (Leica DM IRBE microscope; Leica Microsystems, Vienna, Austria). Sarcomere shortening dynamics were quantified using the IonWizard 6.6 software package (IonOptix, Westwood, MA, USA). CM with a resting sarcomere length (SL) shorter than $1.65 \mu \mathrm{m}$ were excluded from the analyses.

\subsection{Resting Membrane Potential Recordings}

The patch-clamp technique was used to investigate the membrane potential of isolated $\mathrm{CM}$. Experiments were performed at room temperature with a patch-clamp amplifier (200B, Axon Instruments, Foster City, CA, USA) and a Digidata 1440A interface (Axon Instruments). Signals were digitized at $10 \mathrm{kHz}$, low-pass filtered at $1 \mathrm{kHz}$, and analyzed with pCLAMP 10.3 (Axon Instruments) and OriginPro 2021b (OriginLab, Northampton, MA, USA) softwares. Whole-cell recordings in current-clamp mode were obtained with the following bath solution (in $\mathrm{mM}$ ): $140 \mathrm{NaCl}, 5.4 \mathrm{KCl}, 1 \mathrm{CaCl}_{2}, 2 \mathrm{MgCl}_{2}, 10$ glucose, 10 HEPES; pH 7.4, 300 mOsm. The pipette solution contained (in $\mathrm{mM}$ ): $50 \mathrm{KCl}, 80 \mathrm{~K}$ aspartate, $2 \mathrm{MgCl}_{2}$, $3 \mathrm{Mg}$-ATP, 10 EGTA, 10 HEPES; pH 7.4, $300 \mathrm{mOsm}$, as used in previous work [15]. Membrane potential values are given after correction for liquid junction potential. All membrane potentials were recorded at steady state, within 3-5 min after obtaining the seal. In all cases, immediately after obtaining the whole-cell configuration the membrane potential was negative and stable. 


\subsection{RNA Isolation, Reverse Transcription, and Quantitative PCR}

RNA isolation was performed using the RNeasy Mini Kit (Qiagen, Venlo, The Netherlands). CM pellets were lysed immediately following isolation in buffer RLT (provided with the kit) supplemented with $1 \% \beta$-mercaptoethanol. The lysate was processed through QIAshredder columns, and RNA isolated as per the manufacturer's instructions. RNA was eluted in water, and quantity and quality were assessed spectrometrically. cDNA conversion was carried out using TaqMan Reverse Transcription Reagents (ThermoFisher Scientific Inc., Waltham, MA, USA) as per the manufacturer's instructions, using $200 \mathrm{ng}$ of RNA per reaction. Quantitative PCR (qPCR) was conducted using TaqMan pre-designed gene expression assays, Fast Advanced Master Mix (ThermoFisher Scientific Inc., Waltham, MA, USA), and a LightCycler 480 thermocycler (Roche, Pleasanton, CA, USA). Expression levels of voltage-dependent L-type calcium channel (LTCC) $\alpha 2 / \delta$ subunit 1 (CACNA2D1, Cacna2d1, assay ID Oc03397798_m1), $\mathrm{Na}^{+} / \mathrm{Ca}^{2+}$ exchanger (NCX1, Slc8a1, assay ID Oc04250277_m1), and myosin heavy chain- $\beta$ (MHC- $\beta$, Myh7, assay ID Oc03396451_m1) were assessed, and are expressed relative to the levels of mRNA encoding $\beta$-actin (Actb assay ID Oc03824857_g1).

\subsection{Data Analysis}

Data obtained from the same heart are indicated by color and presented as either individual points or as violin superplots including information on data heterogeneitythe normalized density estimates of individual replicates are stacked to show how each replicate (color-coded area) contributes to the overall density estimate (outline) [16]; all graphs additionally indicate the mean \pm standard error of the mean (SEM). Statistical significance of data was assessed based on mean values of all daily recordings to avoid pseudo-replication. Data were analyzed using a paired one-way ANOVA with Tukey's post-hoc test (unless stated otherwise). $p<0.05$ was taken to indicate a statistically significant difference between means.

\section{Results}

\subsection{Yield of Calcium-Tolerant CM over Three Consecutive Days}

Comparisons of the yields of rod-shaped and of regularly contracting (in response to field stimulation) ventricular CM revealed no statistically significant differences between days 0 (heart excision), 1, and 2. The fractions of rod-shaped CM and of CM responding with visible contractions to electrical field stimulation were $\approx 60 \%$ and $\approx 50 \%$, respectively, on all three days (Figure 2A,D). Similarly, the yields of rod-shaped and contracting atrial CM, both from the free wall and from the appendage, did not show statistically significant differences between isolation days, with a yield of $\approx 50 \%$ rod-shaped and of $\approx 40 \%$ contracting CM on all three days (Figure 2B,C,E). There were no statistically significant differences between yields obtained from atrial free wall and appendage.

\subsection{Preservation of CM Morphology over Three Consecutive Days}

Diastolic SL in CM contracting upon stimulation did not show statistically significant differences between isolations on consecutive days, with the mean SL > $1.7 \mu \mathrm{m}$ both in ventricular and in atrial free wall and appendage CM (Figure 3A,C). Qualitative visual examination of both ventricular and atrial CM revealed regular cross-striations and a lack of membrane 'blebbing' in all rod-shaped cells, with no apparent morphological differences between CM isolated on three consecutive days from the same heart (Figure 3B,D and Figure S1 in Supplementary Materials). 

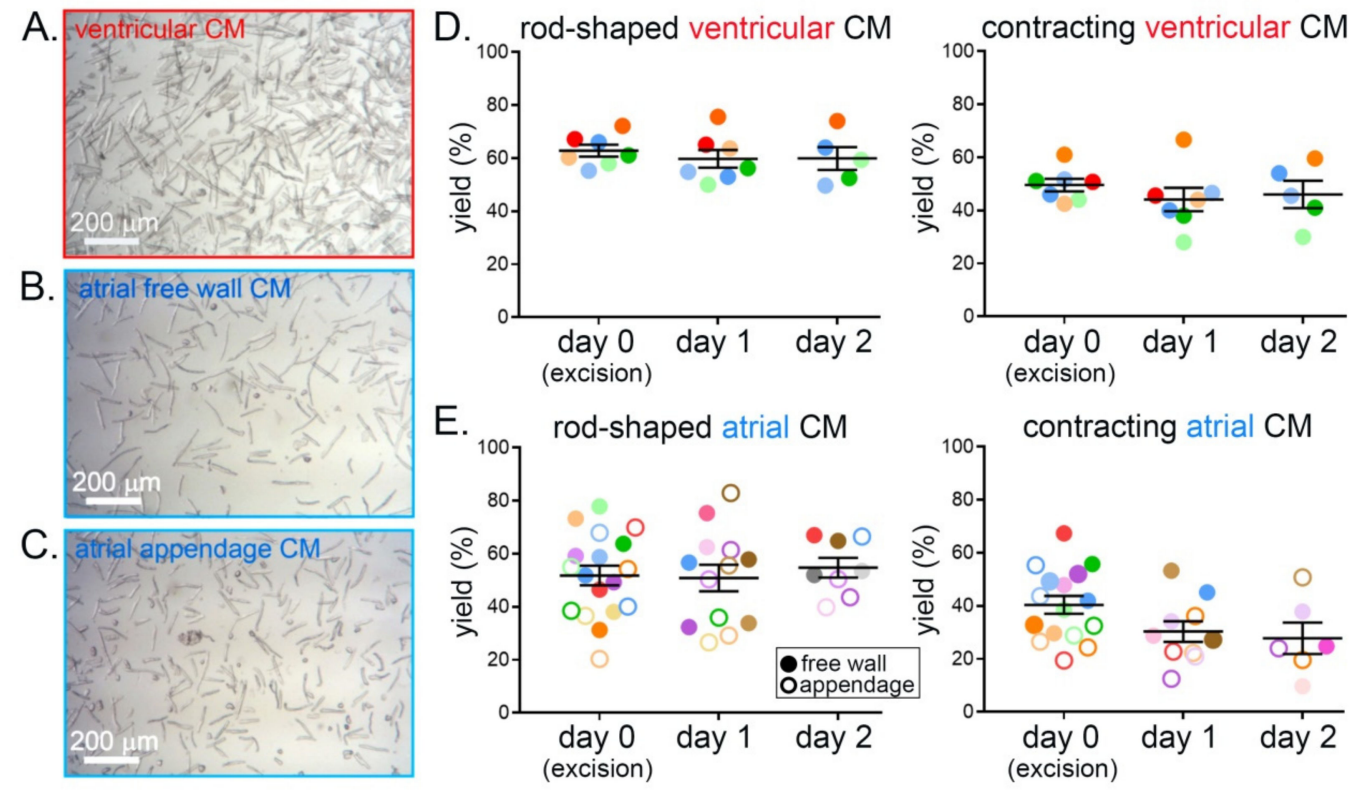

Figure 2. Yield of calcium-tolerant rabbit ventricular and atrial CM does not show statistically significant differences on three consecutive days of cell isolation from the same heart. (A) Representative brightfield microscopy image of ventricular CM after isolation. (B) Representative brightfield microscopy image of atrial free wall CM after isolation. (C) Representative brightfield microscopy image of atrial appendage CM after isolation. (D) Yield (percentage of all cells in the field of view) of rod-shaped and of contracting (upon stimulation; stimulation rate $0.5 \mathrm{~Hz}$ ) ventricular $\mathrm{CM}$ on three consecutive days of isolation from tissue slices of the same heart; $n=5-7$ hearts, $13,000-30,000 \mathrm{CM} /$ day of isolation. (E) Yield of rod-shaped and of contracting atrial CM (stimulation at $1 \mathrm{~Hz}$; free wallfilled circles; appendage-open circles); $n=3-10$ hearts, $800-5700 \mathrm{CM} /$ day of isolation. Each color represents data from one heart.

\subsection{Contraction and Relaxation over Three Consecutive Days}

No statistically significant differences were observed in the degree of sarcomere shortening (Figure 4A,B), or maximum velocity of contraction or relaxation (Figure 4C) of ventricular CM isolated over three consecutive days from tissue slices from the same heart.

Similarly, for atrial CM (both from free wall and appendage), no statistically significant differences were observed with respect to the degree of SL shortening (Figure 5A,B), or maximum velocity of relaxation (Figure $5 \mathrm{C}$ ) over three consecutive days of isolation. However, the maximum velocity of contraction on day 2 was lower than on day 0 (organ excision; Figure 5C). No statistically significant differences were found in the contractile parameters between the different regions of the atria. 

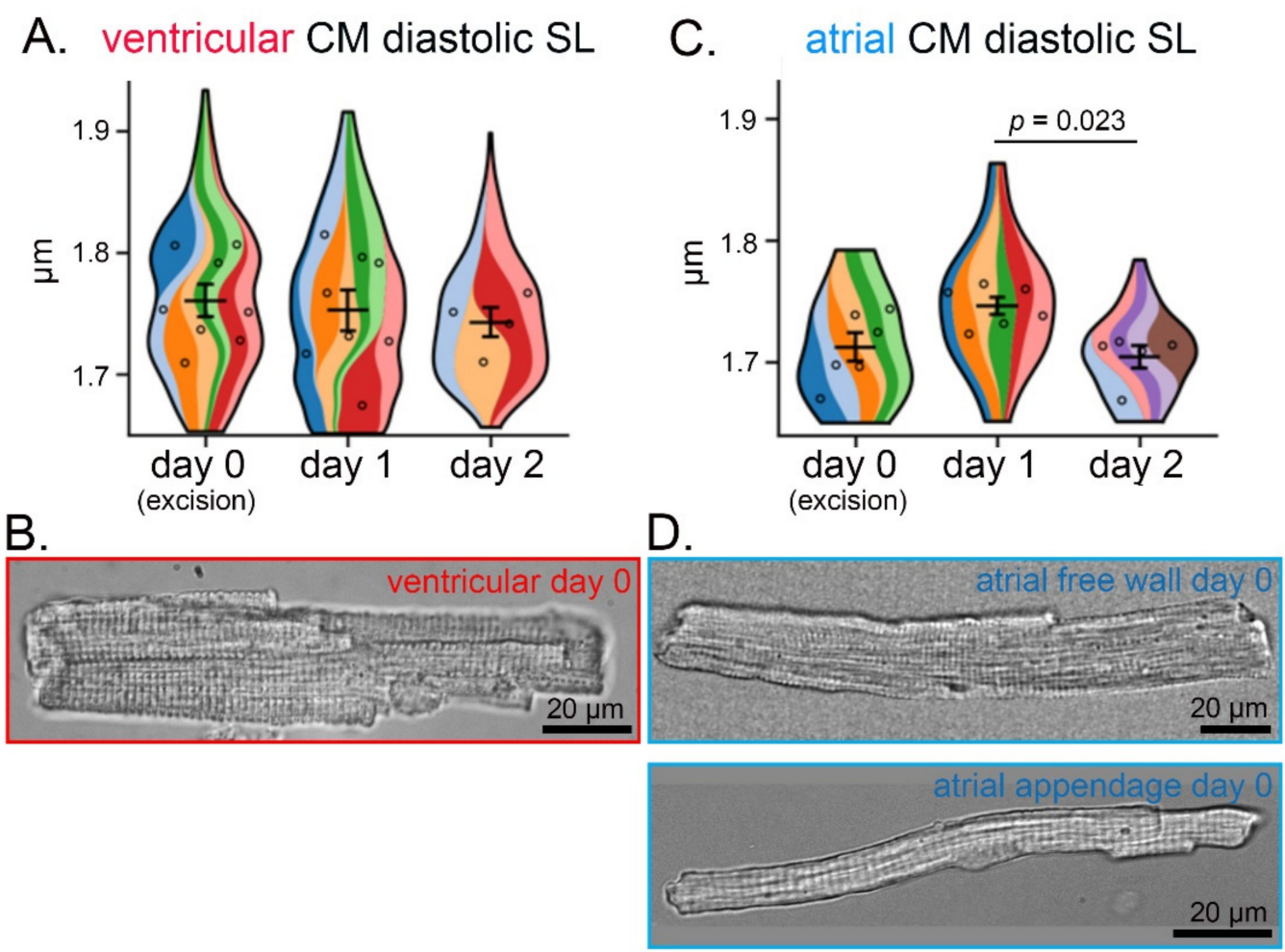

Figure 3. Ventricular and atrial CM morphology does not show statistically significant differences over three consecutive days of cell isolation from cardiac tissue slices from the same rabbit heart. (A) Diastolic SL of isolated ventricular CM over three consecutive days of isolation; $n=4-8$ hearts, 150-300 CM/day of isolation. (B) Representative phase-contrast microscopy image of living ventricular CM after isolation. (C) Diastolic SL of isolated atrial (free wall and appendage combined) CM over three consecutive days of isolation; $n=5-6$ hearts, $40-140 \mathrm{CM} /$ day of isolation. (D) Representative brightfield microscopy image of living atrial free wall (top) and appendage (bottom) CM after isolation. $(\mathbf{A}, \mathbf{C})$ each color represents data from one heart. Within each colored area, the open circles represent the average of all cells recorded from the same heart.

\subsection{Resting Membrane Potential over Three Consecutive Days}

Resting membrane potential was measured using the patch-clamp technique and was found to not differ significantly between ventricular or atrial CM obtained over three consecutive days of isolation from the same heart (Figure 6). 
A. ventricular $\mathrm{CM}$
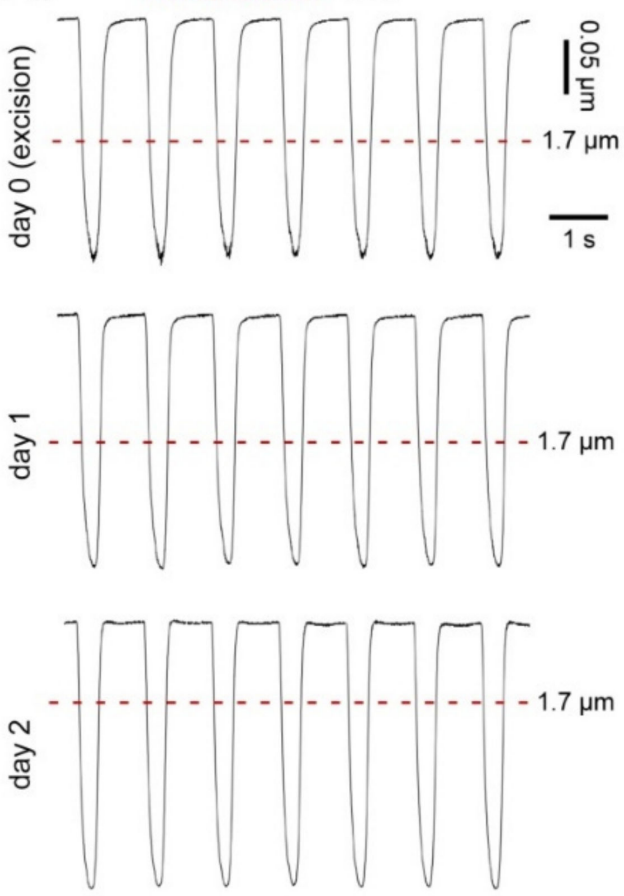

B. ventricular $\mathrm{CM}$ sarcomere shortening

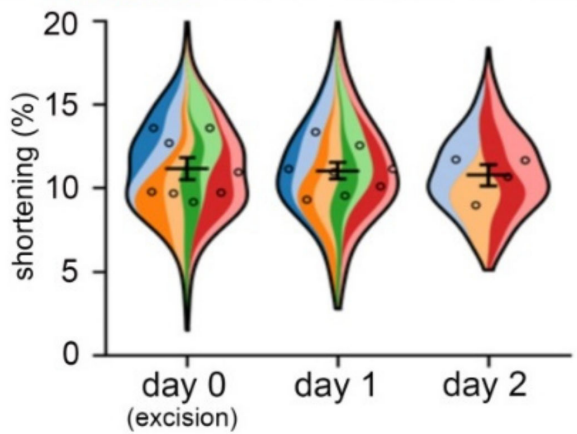

C. ventricular $\mathrm{CM}$ contraction and relaxation

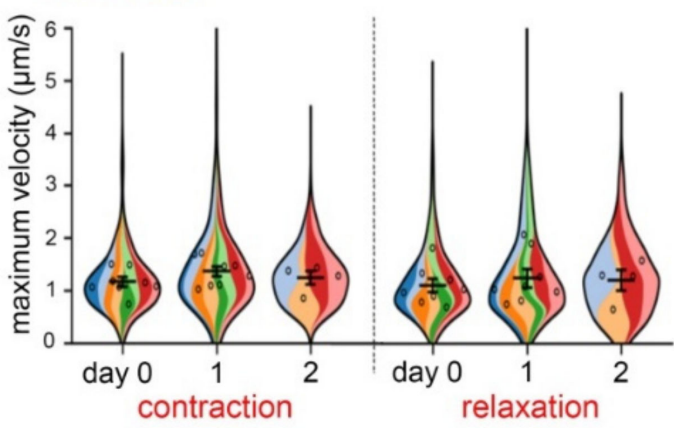

Figure 4. Contraction and relaxation of ventricular $\mathrm{CM}$ over three consecutive days of cell isolation from live cardiac tissue slices of the same heart. (A) Recordings of sarcomere shortening (in response to field stimulation at $0.5 \mathrm{~Hz}$ ) over three consecutive days of CM isolation. (B) Degree of sarcomere shortening (\%) in ventricular CM over three consecutive days. (C) Maximum velocity of contraction and relaxation in ventricular $\mathrm{CM}$ over three consecutive days of $\mathrm{CM}$ isolation; $n=4-8$ hearts, 150-300 CM/day of isolation. (B,C), each color represents data from one heart. Within each colored area, the open circles represent the average of all cells recorded from the same heart.

\subsection{Gene Expression Analysis over Three Consecutive Days}

Transcript level analysis of genes involved in transmembrane calcium movement (encoding L-type $\mathrm{Ca}^{2+}$ channel $\alpha 2 / \delta$ subunit 1 and $\mathrm{Na}^{+} / \mathrm{Ca}^{2+}$ exchanger), as well as in sarcomere integrity (myosin heavy chain- $\beta$ ) of ventricular CM revealed no statistically significant differences between three consecutive days of isolation (Figure 7). These genes were chosen as representative of both functional and structural characteristics of the CM. 


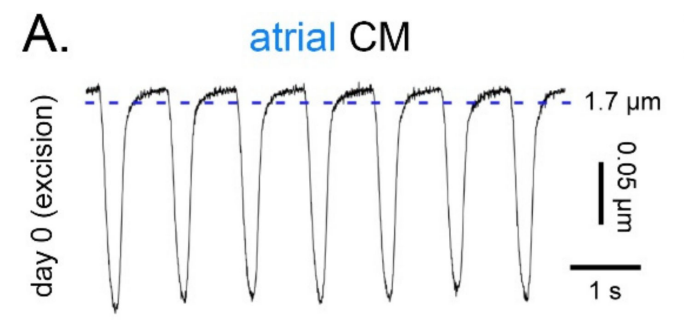

\section{B. atrial $\mathrm{CM}$ sarcomere shortening}

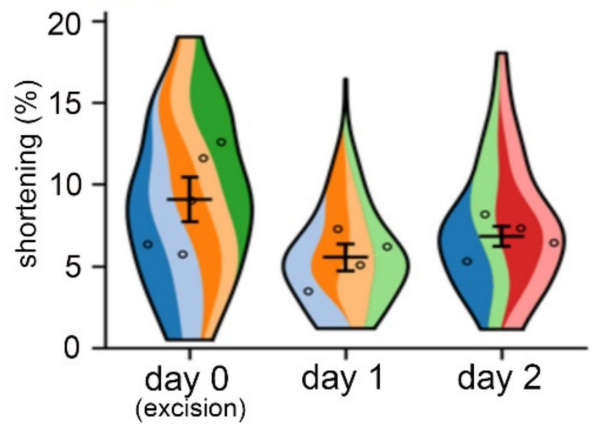

C. atrial $\mathrm{CM}$ contraction and relaxation

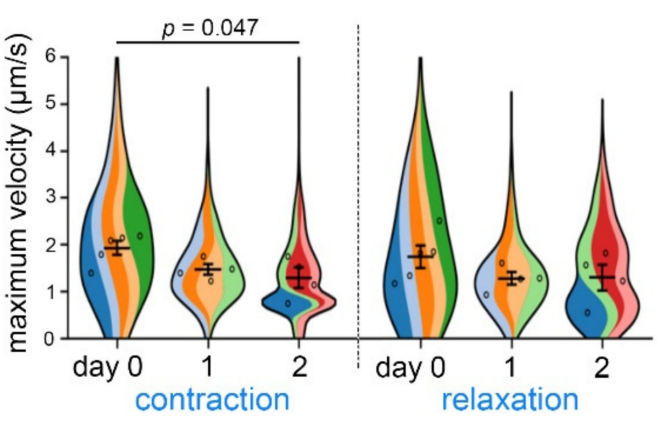

Figure 5. Contraction and relaxation of atrial $\mathrm{CM}$ over three consecutive days of cell isolation from live cardiac tissue slices of the same heart. (A) Recordings of sarcomere shortening (in response to field stimulation at $1 \mathrm{~Hz}$ ) over three consecutive days of $\mathrm{CM}$ isolation from the same heart. (B) Degree of sarcomere shortening (\%) in atrial CM (free wall and appendage combined) over three days of CM isolation. (C) Maximum velocity of contraction and relaxation in atrial CM (free wall and appendage combined) over three consecutive days of CM isolation; $n=4-5$ hearts, $40-70 \mathrm{CM} /$ day of isolation. $(B, C)$, each color represents data from one heart. Within each colored area, the open circles represent the average of all cells recorded from the same heart.

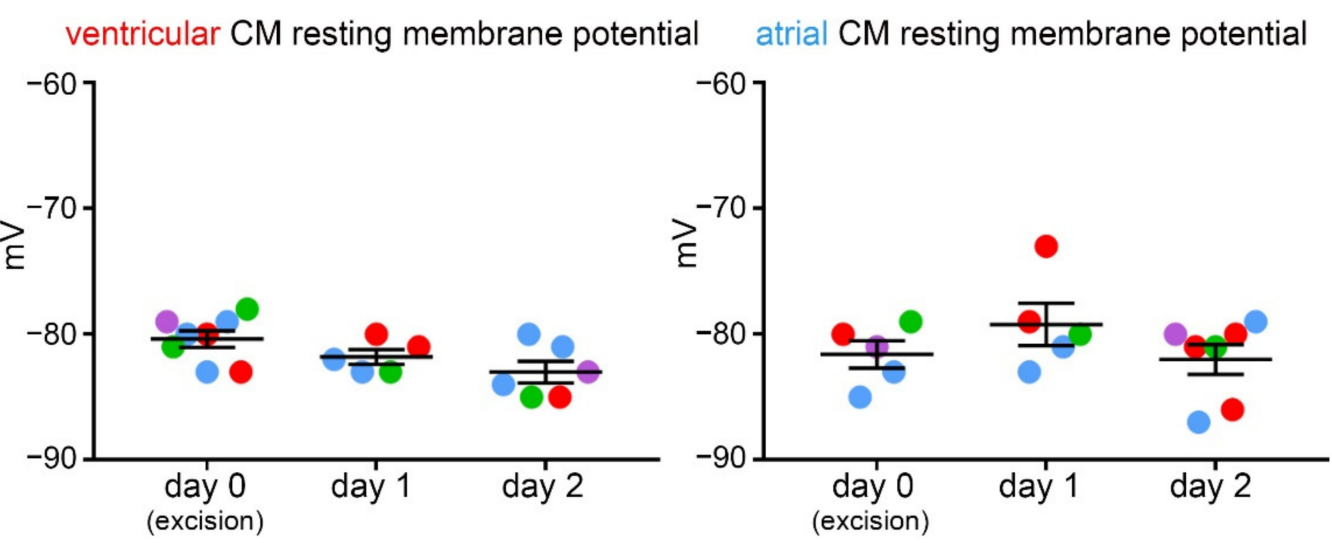

Figure 6. Resting membrane potential in ventricular and atrial CM (free wall and appendage combined) over three consecutive days of cell isolation from live cardiac tissue slices of the same heart; $n=5-8 \mathrm{CM}$ /day of isolation from 3-4 hearts. Each color represents data from one heart. 

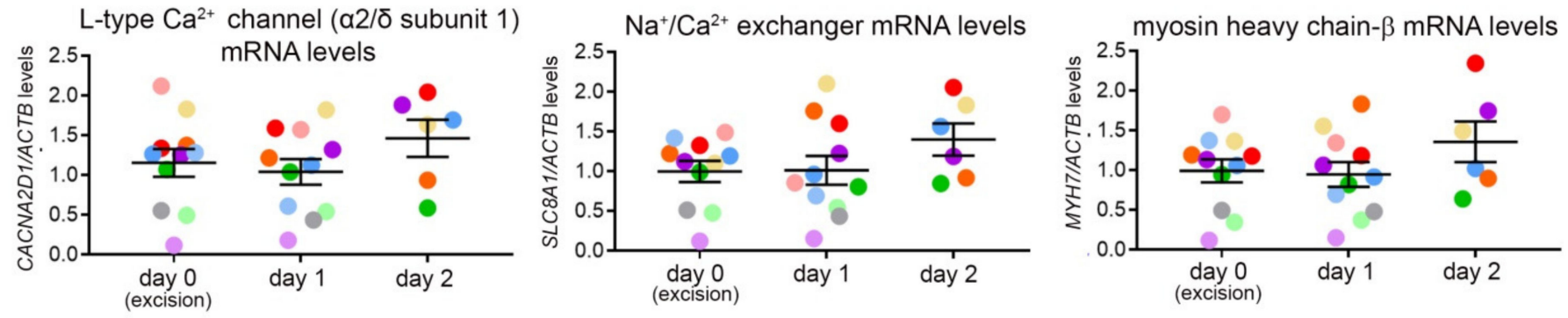

Figure 7. Ventricular $\mathrm{CM}$ gene expression over three consecutive days of cell isolation from live cardiac tissue slices of the same heart. Levels of mRNA, encoding L-type $\mathrm{Ca}^{2+}$ channel $\alpha 2 / \delta$ subunit $1, \mathrm{Na}^{+} / \mathrm{Ca}^{2+}$ exchanger, and myosin heavy chain- $\beta$, over three consecutive days of ventricular $\mathrm{CM}$ isolation. mRNA levels of the genes of interest are expressed relative to the level mRNA encoding $\beta$-actin (control transcript); $n=6-11$ hearts/day of isolation. Each colored dot represents the average of data from the same heart.

\section{Discussion}

Here we present a method of extending the use of cardiac tissue for the isolation of primary ventricular and atrial CM past the day of organ excision. We demonstrate preservation of structural and functional properties of CM over three consecutive days of isolation from the same heart, thus allowing for reduction in experimental animal numbers in basic cardiac research.

We focus our method on rabbit $\mathrm{CM}$, a model whose popularity in basic cardiac research is rapidly rising due to the similarities between rabbit and human cardiac architecture and electrophysiology [17]. To date, however, relatively few rabbit-specific experimental protocols have been made available. Using the method described here, functional CM can be isolated over multiple days following organ excision (currently three), allowing for multiple-day experiments using the same heart. The key advantage of using live tissue as a 'cell micro-environmental incubator' is the maintenance of a close-to-physiological structural micro-environment, including support of the mechanical load. In addition, the use of mechanical uncouplers helps to prevent tissue hypercontracture and preserve the ATP pool during cold storage [18], a concept similar to 'cold cardioplegia' used in transplantology [19-21].

In our study, we used BDM to mechanically uncouple the CM. Our choice was based on previous studies demonstrating positive effects of BDM on the viability of live cardiac tissue slices and isolated CM [22-25]. However, while BDM has been shown to improve preservation of $\mathrm{CM}$ contractility in comparison to blebbistatin, blebbistatin was shown to better preserve $\mathrm{CM}$ viability (defined as maintenance of rod-shaped morphology) [22]. In addition, BDM was suggested to have negative effects on mitochondrial respiration [26]. Therefore, the choice of the most suitable approach for future applications will depend on the individual research questions. As an additional alternative, N-benzyl-p-toluene sulphonamide (BTS) has been shown previously to extend the viability of single isolated canine CM [27].

In terms of $\mathrm{CM}$ quality assessment, the work presented here focuses on gross morphology, diastolic SL, contraction/relaxation kinetics, resting membrane potential, and transcript levels of several selected genes. A future in-depth analysis of $\mathrm{CM}$ mechanics (e.g., force generation), calcium handling mechanisms, $\mathrm{CM}$ metabolic profile and morphology (e.g., of transverse tubules or sarcomeres), as well as of large-scale gene expression and proteome profiles will be important to further understand the degree of CM preservation. In addition, prolonging the protocol to include further consecutive days is a goal, which may further increase the relevance of the method described here.

Crucially, the majority of rod-shaped CM-about $50 \%$ of ventricular and $40 \%$ of atrial $\mathrm{CM}$-responded to field stimulation. Multi-day isolation of ventricular $\mathrm{CM}$ yielded structurally and functionally consistent cell populations. In the case of atrial $\mathrm{CM}$, a reduced 
contraction velocity was observed on day 2. Atrial CM, however, exhibited no deterioration in any of the other parameters assessed. The reasons for a decline in atrial CM contraction velocity are currently unclear. It is possible that slight differences in storage (slices vs. chunks), isolation (duration of digestion) or recording protocols underlie these observations. Another possibility is that atrial CM are more susceptible to damage [28]. Further investigation of electrophysiological properties and an assessment of calcium handling mechanisms in atrial CM would help identify the most likely cause for the decline in contraction velocity seen on day 2 post-isolation. Refinement of the described protocol (also with other species in mind) may include the composition of solutions and specific CM isolation workflows for different heart regions. One possibility is the employment of the University of Wisconsin solution as a storage buffer-it was previously reported to successfully preserve both ventricular and atrial human tissue, and is thought to offer more precise control of osmotic and oncotic pressure, redox homeostasis, and metabolic turnover [29,30]. Alternatively, low sodium/high potassium solutions could be employed to prevent tissue hypercontracture during prolonged storage. In addition, a host of supplements have been previously suggested to enhance the survival of cultured CM-thyroid hormones, insulin, L-glutamate, creatine, L-carnitine, and taurine [21,31]. Finally, the viability of slices could be extended by culturing under constant electro-mechanical stimulation (shown to preserve slice function) between isolations [12,32-34].

Although slice-based isolation methods do not reach the yield obtained with coronary artery perfusion-based isolation methods [13], they provide multiple advantages. With a slice-based method, a clear separation between regions of interest is easily possible. Here, we separated the ventricles, atrial free wall, and appendage. Other conceivable applications include the investigation of remodeled vs. non-remodeled myocardium post-injury or sub-epicardial vs. sub-endocardial CM. Furthermore, slice-based methods can be adapted for multiple species, including humans, where coronary perfusion with enzyme-containing solution is usually not possible [13]. Finally, the adaptation of the slice-based isolation protocol to new species and tissues is easier compared to Langendorff based methods, allowing for multiple parallel and cost-saving comparisons of different experimental conditions (e.g., choice of enzyme, solution composition) using multiple fragments or slices from the same heart. Slice-based isolation methods would, however, be of limited use when larger numbers of CM are required (e.g., for membrane fractionation experiments or high-throughput drug testing), and they are also in general more timeconsuming compared to Langendorff based isolation.

The major advantage of the presented method is its simplicity and ease of implementation in any laboratory. We believe that further development of the method will help researchers arrive at a 'one-heart-per-working-week' paradigm, instead of the current one-heart-per-experimental-day approach. This will save costs and lead to a significant reduction in the number of experimental animals, supporting the guiding principle of 3R (replacement, reduction, refinement).

Supplementary Materials: The following are available online at https:/ /www.mdpi.com/article/10 .3390 /cells11020233/s1, Table S1: Composition of solutions, Figure S1: Representative brightfield and phase-contrast microscopy images of living ventricular and atrial CM after isolation at day 0, 1 and 2. All scale bars correspond to $20 \mu \mathrm{m}$, atrial cells at day 1 and 2 are phase-contrast microscopy images. The regions of interest used to record SL are shown in magenta.

Author Contributions: Conceptualization, E.A.R.-Z., R.P., J.G., T.S. (Teresa Schiatti); methodology, J.G., T.S. (Teresa Schiatti), T.S. (Thomas Seidel), W.K., M.D., A.S., T.K., D.J.F., data acquisition and analysis, J.G., T.S. (Teresa Schiatti), T.S. (Thomas Seidel), W.K., M.D., A.S., T.K., D.J.F., E.A.R.-Z., R.P.; writing-review and editing, E.A.R.-Z., R.P., J.G., T.S. (Teresa Schiatti), T.S., P.K.; supervision, T.S. (Thomas Seidel), U.R., P.K., R.P. and E.A.R.-Z.; funding acquisition, T.S., U.R., P.K., E.A.R.-Z. All authors have read and agreed to the published version of the manuscript.

Funding: This research was supported by the German Research Foundation Emmy Noether Programme (396913060 to E.A.R.-Z.) and by the European Union's Horizon 2020 research and innovation 
programme (under the Marie Skłodowska-Curie grant agreement (860974 to T.S. (Teresa Schiatti)), U.R., and R.P.). J.G., T.S. (Thomas Seidel), U.R., P.K., R.P., and E.A.R.-Z. are members of the Collaborative Research Centre SFB1425 of the German Research Foundation (422681845).

Institutional Review Board Statement: The study was conducted according to the guidelines stated in Directive 2010/63/EU of the European Parliament on the protection of animals used for scientific purposes and approved by the local authorities in Baden-Württemberg (X-16/10R).

Informed Consent Statement: Not applicable.

Data Availability Statement: Data is available from the corresponding author upon request.

Acknowledgments: We would like to thank Simone Nübling, Stefanie Perez-Feliz, Cinthia Walz, Pia Iaconniani, Kristina Kollmar, Sophie Pilgrim, Sofía Orós Rodrigo, and Jiaying Fu for technical assistance. Imaging data were obtained with the help of the SCI-MED facility at the Institute for Experimental Cardiovascular Medicine, University Heart Centre Freiburg/Bad Krozingen. We also thank the Lighthouse Facility of the University of Freiburg for providing access to a transcript analysis platform. We thank Clemens Kreutz (Institute of Medical Biometry and Statistics, University of Freiburg) for statistical advice.

Conflicts of Interest: The authors declare no conflict of interest.

\section{References}

1. Gould, R.P.; Powell, T. Intact isolated muscle cells from the adult rat heart. J. Physiol. 1972, 225, 16-19.

2. Jacobson, S.L.; Piper, H.M. Cell cultures of adult cardiomyocytes as models of the myocardium. J. Mol. Cell Cardiol. 1986, 18, 661-678. [CrossRef]

3. Mitcheson, J.S.; Hancox, J.C.; Levi, A.J. Cultured adult cardiac myocytes: Future applications, culture methods, morphological and electrophysiological properties. Cardiovasc. Res. 1998, 39, 280-300. [CrossRef]

4. Volz, A.; Piper, H.M.; Siegmund, B.; Schwartz, P. Longevity of adult ventricular rat heart muscle cells in serum-free primary culture. J. Mol. Cell Cardiol. 1991, 23, 161-173. [CrossRef]

5. Burton, R.A.B.; Rog-Zielinska, E.A.; Corbett, A.D.; Peyronnet, R.; Bodi, I.; Fink, M.; Sheldon, J.; Hoenger, A.; Calaghan, S.C.; Bub, G.; et al. Caveolae in rabbit ventricular myocytes: Distribution and dynamic diminution after cell isolation. Biophys. J. 2017, 113, 1047-1059. [CrossRef]

6. Hammer, K.; Ruppenthal, S.; Viero, C.; Scholz, A.; Edelmann, L.; Kaestner, L.; Lipp, P. Remodelling of Ca ${ }^{2+}$ handling organelles in adult rat ventricular myocytes during long term culture. J. Mol. Cell Cardiol. 2010, 49, 427-437. [CrossRef]

7. Mitcheson, J.S.; Hancox, J.C.; Levi, A.J. Action potentials, ion channel currents and transverse tubule density in adult rabbit ventricular myocytes maintained for 6 days in cell culture. Pflug. Arch. 1996, 431, 814-827. [CrossRef]

8. Himmel, H.M.; Pietsch, M.; Streller, U.; Graf, E.M.; Ravens, U. Changes in morphology and inward rectifier currents in human atrial myocytes depend on culture conditions. Basic Res. Cardiol. 2002, 97, 434-444. [CrossRef]

9. $\quad$ Ellingsen, O.; Davidoff, A.J.; Prasad, S.K.; Berger, H.-J.; Springhorn, J.P.; Marsh, J.D.; Kelly, R.A.; Smith, T.W. Adult rat ventricular myocytes cultured in defined medium: Phenotype and electromechanical function. Am. J. Physiol. Heart Circ. Physiol. 1993, 265, 747-754. [CrossRef]

10. Laird, D.W.; Puranam, K.L.; Revel, J.P. Turnover and phosphorylation dynamics of connexin-43 gap junction protein in cultured cardiac myocytes. Biochem. J. 1991, 273, 67-72. [CrossRef]

11. Louch, W.E.; Sheehan, K.A.; Wolska, B.M. Methods in cardiomyocyte isolation, culture, and gene transfer. J. Mol. Cell Cardiol. 2011, 51, 288-298. [CrossRef]

12. Fischer, C.; Milting, H.; Fein, E.; Reiser, E.; Lu, K.; Seidel, T.; Schinner, C.; Schwarzmayr, T.; Schramm, R.; Tomasi, R.; et al. Long-term functional and structural preservation of precision-cut human myocardium under continuous electromechanical stimulation in vitro. Nat. Commun. 2019, 10, 117. [CrossRef]

13. Fiegle, D.J.; Volk, T.; Seidel, T. Isolation of human ventricular cardiomyocytes from vibratome-cut myocardial slices. J. Vis. Exp. 2020, 159, e61167. [CrossRef]

14. Peyronnet, R.; Bollensdorff, C.; Capel, R.A.; Rog-Zielinska, E.A.; Woods, C.E.; Charo, D.N.; Lookin, O.; Fajardo, G.; Ho, M.; Quertermous, T.; et al. Load-dependent effects of apelin on murine cardiomyocytes. Prog. Biophys. Mol. Biol. 2017, 130, 333-343. [CrossRef] [PubMed]

15. Bernal Sierra, Y.A.; Rost, B.R.; Pofahl, M.; Fernandes, A.M.; Kopton, R.A.; Moser, S.; Holtkamp, D.; Masala, N.; Beed, P.; Tukker, J.J.; et al. Potassium channel-based optogenetic silencing. Nat. Commun. 2018, 9, 4611. [CrossRef]

16. Kenny, M.; Schoen, I. Violin SuperPlots: Visualizing replicate heterogeneity in large data sets. Mol. Biol. Cell 2021, 32, 1333-1334. [CrossRef]

17. Odening, K.E.; Kohl, P. Follow the white rabbit: Experimental and computational models of the rabbit heart provide insights into cardiac (patho-) physiology. Prog. Biophys. Mol. Biol. 2016, 121, 75-76. [CrossRef] 
18. Hebisch, S.; Bischoff, E.; Soboll, S. Influence of 2,3-butanedione monoxime on heart energy metabolism. Basic Res. Cardiol. 1993, 88, 566-575. [CrossRef]

19. Lee, K.C.; Chang, C.Y.; Chuang, Y.C.; Sue, S.H.; Yang, H.S.; Weng, C.F.; Lee, Y.T.; Huang, W.S.; Chen, I.C.; Wei, J. Combined St. Thomas and histidine-tryptophan-ketoglutarat solutions for myocardial preservation in heart transplantation patients. Transpl. Proc. 2012, 44, 886-889. [CrossRef]

20. Jahania, M.S.; Sanchez, J.A.; Narayan, P.; Lasley, R.D.; Mentzer, R.M., Jr. Heart preservation for transplantation: Principles and strategies. Ann. Thorac. Surg. 1999, 68, 1983-1987. [CrossRef]

21. Gharagozloo, F.; Melendez, F.J.; Hein, R.A.; Laurence, R.G.; Shemin, R.J.; DiSesa, V.J.; Cohn, L.H. The effect of amino acid L-glutamate on the extended preservation ex vivo of the heart for transplantation. Circulation 1987, 76, 65-70.

22. Chung, C.S.; Mechas, C.; Campbell, K.S. Myocyte contractility can be maintained by storing cells with the myosin ATPase inhibitor 2,3 butanedione monoxime. Physiol. Rep. 2015, 3, e12445. [CrossRef] [PubMed]

23. Watson, S.A.; Scigliano, M.; Bardi, I.; Ascione, R.; Terracciano, C.M.; Perbellini, F. Preparation of viable adult ventricular myocardial slices from large and small mammals. Nat. Protoc. 2017, 12, 2623-2639. [CrossRef] [PubMed]

24. Kivisto, T.; Makiranta, M.; Oikarinen, E.L.; Karhu, S.; Weckstrom, M.; Sellin, L.C. 2,3-butanedione monoxime (BDM) increases initial yields and improves long-term survival of isolated cardiac myocytes. Jpn. J. Physiol. 1995, 45, 203-210. [CrossRef] [PubMed]

25. Mulieri, L.A.; Hasenfuss, G.; Ittleman, F.; Blanchard, E.M.; Alpert, N.R. Protection of human left ventricular myocardium from cutting injury with 2,3-butanedione monoxime. Circ. Res. 1989, 65, 1441-1449. [CrossRef]

26. Hall, A.R.; Hausenloy, D.J. Mitochondrial respiratory inhibition by 2,3-butanedione monoxime (BDM): Implications for culturing isolated mouse ventricular cardiomyocytes. Physiol. Rep. 2016, 4, e12606. [CrossRef]

27. Abi-Gerges, N.; Pointon, A.; Pullen, G.F.; Morton, M.J.; Oldman, K.L.; Armstrong, D.; Valentin, J.P.; Pollard, C.E. Preservation of cardiomyocytes from the adult heart. J. Mol. Cell Cardiol. 2013, 64, 108-119. [CrossRef]

28. Brandenburg, S.; Arakel, E.C.; Schwappach, B.; Lehnart, S.E. The molecular and functional identities of atrial cardiomyocytes in health and disease. Biochim. Biophys. Acta Mol. Cell Res. 2016, 1863, 1882-1893. [CrossRef]

29. Guo, G.-R.; Chen, L.; Rao, M.; Chen, K.; Song, J.P.; Hu, S.S. A modified method for isolation of human cardiomyocytes to model cardiac diseases. J. Transl. Med. 2018, 16, 288. [CrossRef]

30. Stringham, J.C.; Paulsen, K.L.; Southard, J.H.; Mentzer, R.M., Jr.; Belzer, F.O. Forty-hour preservation of the rabbit heart: Optimal osmolarity, $\left[\mathrm{Mg}^{2+}\right]$, and pH of a modified UW solution. Ann. Thorac. Surg. 1994, 58, 7-13. [CrossRef]

31. Mitcheson, J.S.; Hancox, J.C.; Levi, A.J. Cultured adult rabbit myocytes: Effect of adding supplements to the medium, and response to isoprenaline. J. Cardiovasc. Electrophysiol. 1997, 8, 1020-1030. [CrossRef]

32. Meki, M.H.; Miller, J.M.; Mohamed, T.M.A. Heart slices to model cardiac physiology. Front. Pharmacol. 2021, 12, 617922. [CrossRef]

33. Berger, H.J.; Prasad, S.K.; Davidoff, A.J.; Pimental, D.; Ellingsen, O.; Marsh, J.D.; Smith, T.W.; Kelly, R.A. Continual electric field stimulation preserves contractile function of adult ventricular myocytes in primary culture. Am. J. Physiol. 1994, 266, 341-349. [CrossRef]

34. Edelmann, J.C.; Jones, L.; Peyronnet, R.; Lu, L.; Kohl, P.; Ravens, U. A Bioreactor to apply multimodal physical stimuli to cultured cells. Methods Mol. Biol. 2016, 1502, 21-33. 